\title{
THE IMPACT OF JEAN-PAUL ZAHN DISCOVERIES ON ROTATION AND EVOLUTION
}

\author{
A. Maeder ${ }^{1}$ and G. Meynet ${ }^{1}$
}

\begin{abstract}
We first review the main effects of stellar rotation on evolution along the fundamental discoveries by Jean-Paul. Then, we examine some of the consequences of rotation in the evolution of single and binary stars. The proper account of meridional circulation in close binaries tends to increase the synchronization time because meridional currents always counteract the tidal interaction. We consider the case of the very low metallicity $Z$ stars, in particular the CEMP-no stars, where rotational mixing may have played a dominant role in their strange chemical composition. Then, turning to "What are the mysteries?", we emphasize that all over the evolution and for various masses the present models seem to still have a lack of rotational coupling between cores and envelopes. We suggest that magnetic fields may produce this missing internal coupling.
\end{abstract}

\section{Introduction: A brief history of rotation}

It is with emotion and respect that we think to our great colleague Jean-Paul Zahn, to whom we are indebted to so much progresses in our understanding of the effects of rotation on stellar evolution, besides his many other major contributions on stellar tides, on stellar oscillations and convection.

His discoveries take a good place in the long History of Stellar Rotation, which started in 1610 with the finding of solar rotation by Galileo Galilei. From the $18^{\text {th }}$ century onwards, the equilibrium figures of rotating bodies were studied by McLaurin, Jacobi, Poincaré, Wavre and others. The bright idea to use the width of the spectral lines was first proposed by de Abney in 1877 and the first effective application to a sample of stars was achieved by Shajn and Struve in 1929. The gaseous rotating stars were studied by Eddington in the 1920's, who pointed out the breakdown of radiative equilibrium in a rotating star. From the 1950's the

\footnotetext{
${ }^{1}$ Department of Astronomy, University of Geneva, Switzerland
} 
circulation currents were studied by Sweet and Roy. A particular emphasis on the effects in the outer stellar layers was brought by Gratton and by Öpik, while the considerable effects of the mean molecular weight were studied by Mestel. From the 1960's, the first numerical models of rotating stars were calculated by Roxburgh, and then by Kippenhahn who emphasized the problem of the conservation of the angular momentum in circulation currents. In 1979, Deubner, Ulrich and Rhodes demonstrated that solar oscillations give us an access to the internal solar rotation, a finding of a large impact in today helio- and asteroseismology. The famous 1992 paper by Jean-Paul (Zahn 1992) is the first consistent theory of meridional circulation with a proper account of the transport of angular momentum and chemical elements. This work forms the strong rock above which all consistent stellar models were constructed in the following decades.

Scientists are also great by the number of their coworkers and followers. In this respect, we note the great number of colleagues, who had the chance to have the illuminating experience to collaborate with Jean-Paul. Many of them are present here.

In Section 2, we briefly summarize the basic ideas governing the physics of rotating stars. In Section 3, the basic effects of rotation in single and binary stars are presented. In Section 4, the case of rotation in the extremely low metallicity stars is examined. Section 5 tries to answer the question "Where are the mysteries?" asked by Juri Toomre to somebody during this meeting. We are finding this question as an essential one because the present mysteries will be the Science of tomorrow. Section 6 gives a brief conclusion.

\section{The basic loop of the physics of rotating models}

The basic ideas and consistent equations of the physics of rotating stars were established by Jean-Paul (Zahn 1992). Let us briefly review the main points of this treatment. The internal rotation is assumed to be shellular, which means that the angular velocity $\Omega$ is considered as constant on equipotentials. One may write

$$
\Omega(r, \vartheta)=\overline{\Omega(r)}+\Omega_{2}(r) P_{2}(\vartheta)
$$

At low and moderate rotation velocities, the equipotentials can be approximated by spherical surfaces, thus the use of the coordinate $r$ in (2.1). Shellular rotation results from the very strong horizontal turbulence, likely dominating in rotating stars. The exact expression of the diffusion coefficient $D_{\mathrm{h}}$ of the horizontal turbulence, which results from horizontal shears on the equipotentials, remains rather uncertain. The one-dimensional equations of stellar structure accounting for shellular rotation have been written by Meynet \& Maeder (1997). They express the effects of the centrifugal force on the average internal distribution of the physical variables $r, T, P, L_{r}$. This last effect is significant, but it is largely dominated by the transports of angular momentum and of chemical elements. Figure 1 illustrates the basic loop governing these effects as shown by Jean-Paul. The velocity $U(r)$ of meridional circulation accounting for shellular rotation contains the driving term 


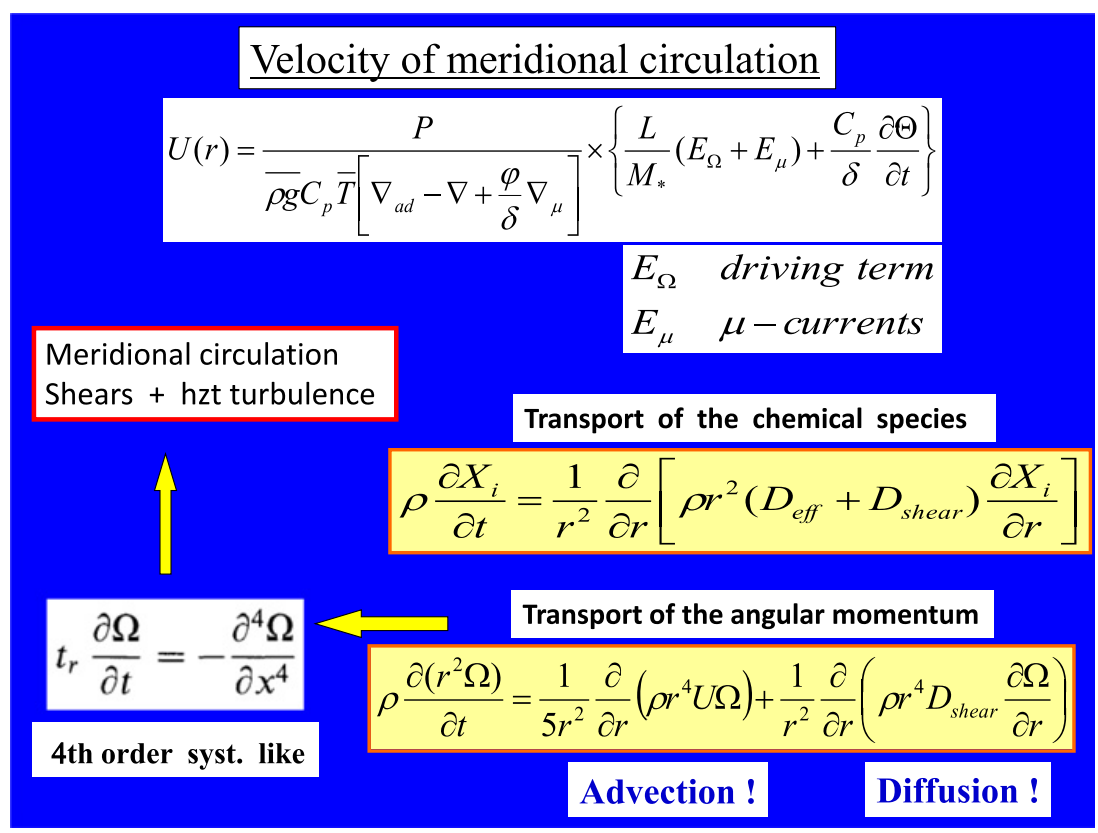

Fig. 1. The basic loop expressing the transport of angular momentum and chemical elements intervening in the construction of models of rotating stars (see text).

$E_{\Omega}$ (Zahn 1992). It depends on the ratio of the centrifugal force to gravity, on the $\Omega$-profile and is also affected by the horizontal turbulence $D_{\mathrm{h}}$ (Chaboyer \& Zahn 1992). The effects of the gradients of mean molecular weight $\mu$ are expressed by the term $E_{\mu}$ (Maeder \& Zahn 1998). The comparison of rotating models from various authors shows that the main source of differences between authors lies in the way mixing is treated and in particular in the inhibiting effects of the $\mu$-gradients.

Rotation drives internal currents and various turbulent instabilities. The main instability contributing to both transports is the shear instability, it results form the velocity differences of adjacent layers. The instability criterion is the Richardson criterion, which says that an instability develops if the excess of energy in differential motions (excess with respect to the energy for an average velocity) is bigger than the work necessary to exchange matter vertically. It also depends on the gradient of composition, as well as on the horizontal turbulence. This is a particularly critical point, since the account of the horizontal turbulence weakens the inhibiting effects of the $\mu$-gradients (Talon \& Zahn 1997). Several other instabilities could also play a role for the transport of chemical elements and angular momentum, in particular the thermohaline mixing, the Rayleigh-Taylor, the Solberg-Hoiland and GSF instabilities. A unified treatment of these various instabilities was proposed by Maeder et al. (2013). We note in particular, that 
the Rayleigh-Taylor instability could intervene in the pre-supernova stages when the $\Omega$-gradient is very steep at the edge of the stellar core.

The instabilities may contribute to the internal transports of the elements and angular momentum. The effects of shears with the diffusion coefficient $D_{\text {shear }}$ are generally considered to dominate. The transport of the chemical elements by the meridional circulation combined with the effects of the strong horizontal turbulence, which tends to homogenize the horizontal layers, is equivalent to a diffusion process with coefficient $D_{\text {eff }}=\left(r U_{2}\right)^{2} /\left(30 D_{\mathrm{h}}\right)$ (Chaboyer \& Zahn 1992), see also Figure 1. While the combined effects of shears and horizontal turbulence have been discussed by Talon \& Zahn (1997).

In a rotating star, the evolution of the angular velocity $\Omega(r, t)$ has to be followed at each level r. We see (Fig. 1) that the equation governing this transport is different from that for the chemical elements (Zahn 1992). The first term on the right is an advective term resulting from the currents of meridional circulation. Ignoring this term or replacing it by a diffusion, as is often made by some authors, is not a consistent choice. For the transport of angular momentum, the local conservation of the angular momentum limits the effects of the horizontal turbulence $D_{\mathrm{h}}$ and it is considered that it does not play a significant role here, contrarily to the case of the chemical elements.

There is a loop of interactions (Fig. 1): circulation and shears influence the transport of the angular momentum, which influences the angular velocity $\Omega(r, t)$. In turn, the distribution of angular velocity determines the shear and the circulation velocity. Simultaneously, the transport of chemical elements modifies the $\mu$-gradients, which also intervene in the coefficients of transport. The consistency of the whole loop has to be treated carefully, in a simplified way it is equivalent to an equation containing a fourth-order space derivative of $\Omega(r, t)$. The time scale of the meridional circulation behaves like the Kelvin-Helmholtz timescale divided by the ratio of the centrifugal force to gravity, so that meridional circulation significantly acts only in long nuclear burning phases.

\section{The effects of rotation in single and binary stars}

Rotation affects all the properties, outputs and observations of stellar evolution (Maeder 2009: lifetimes, tracks in the HRD, binary evolution, asteroseismology, LBV, WR stars and their nebulae, Cepheids, surface chemical abundances, formation of primary N, pre-supernova stages, chemical yields and nucleosynthesis, rotation periods of pulsars, final stellar masses, collapsars, $\gamma$-bursts, first stars in the Universe, etc...

\subsection{Single stars}

The surface enrichments in products of the CNO burning, observable for example by the $\mathrm{N} / \mathrm{H}$, or $\mathrm{N} / \mathrm{C}$ ratios, increase with stellar mass $\mathrm{M}$ and rotation velocities $v$ (Maeder 2009). The enrichments also increase with the age $t$, since more and more new nuclear products reach the stellar surface (Fig. 2). The enrichments 


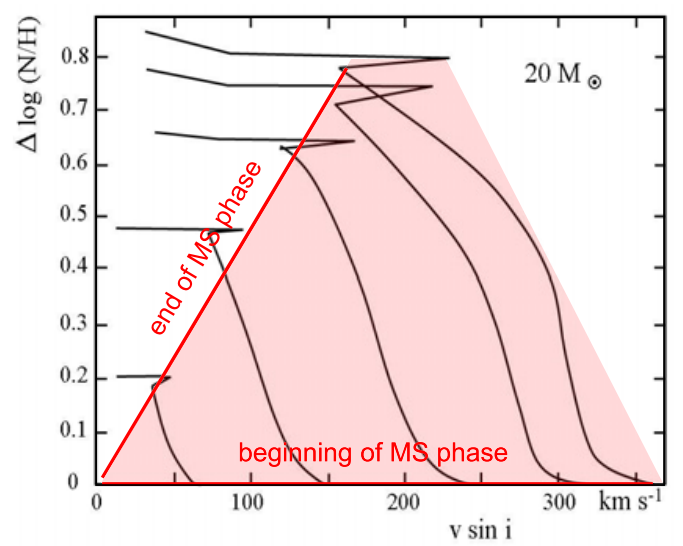

Fig. 2. The changes of the ratios $(N / H)$ (in numbers) and rotation velocities from the zero-age sequence to the end of the main sequence for different initial velocities in the case of a $20 M_{\odot}$ star with $Z=0.02$.

are also stronger at lower $\mathrm{Z}$, shear mixing being favored in more compact stars. Tidal interactions, as seen below, may influence the mixing. Thus, the chemical enrichments are a multivariate function, e.g.,

$$
(N / H)=f(M, v, t, Z, \text { binarity }, \cdots) .
$$

The neglect of the richness of this function by some authors just concentrating on a single relation of the form $(\mathrm{N} / \mathrm{H})$ vs. velocity has led to a decade of useless debates.

The He-enrichments at the surface occur much more slowly than the enrichments in nitrogen, since the internal He-gradient is much weaker that the N-gradient. Careful analyses have well confirmed the predicted effects. A tight trend in the observed $\mathrm{N} / \mathrm{C}$ vs. $\mathrm{N} / \mathrm{O}$ ratios and the build-up of helium is found from main-sequence to supergiant stars (Przybilla et al. 2010). A careful spectroscopic survey of galactic O-stars by Martins et al. (2015) confirms many predicted effects of rotation. Figure 3 well illustrates the age effect on the chemical enrichments. A clear trend of stronger N-enrichments in more evolved stars is observed. The trend of more mixing in more massive objects is present both among dwarfs and supergiants. A fraction of $90 \%$ of the sample stars are well accounted for by the rotational effects predicted by the models of Ekstroem et al. (2012). The evidence of more mixing at lower $\mathrm{Z}$ is supported by the evidences of a production of primary nitrogen in low $\mathrm{Z}$ stars. The observations of the ratio ${ }^{12} \mathrm{C} /{ }^{13} \mathrm{C}$ in low $Z$ stars, as well as other observations (Sect. 4), are also supporting these conclusions, see Maeder \& Meynet (2012). As to the magnetic effects in rotating stars, a variety of N-enrichments is observed in magnetic OB stars (Fig. 3) so that it is unclear for now if mixing is enhanced by magnetic fields or not. 


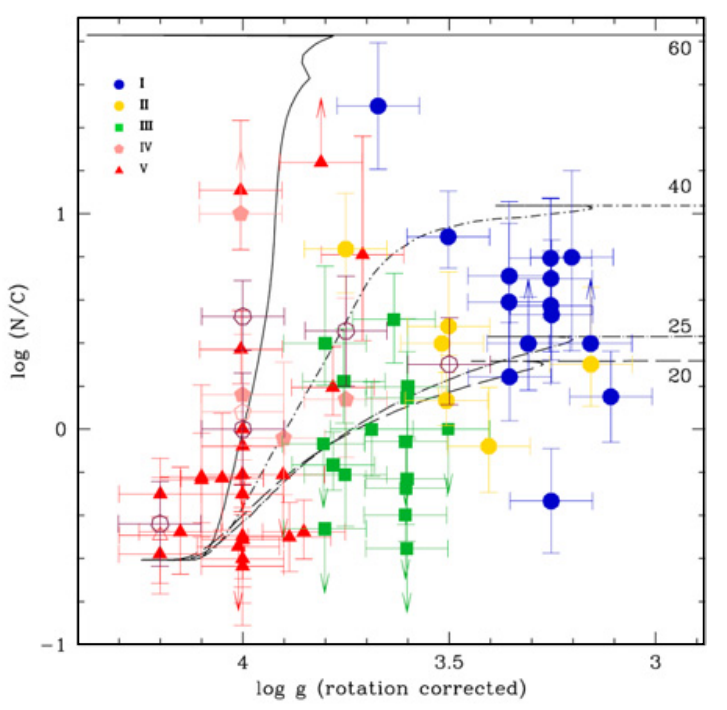

Fig. 3. $\log (\mathrm{N} / \mathrm{C})$ vs. $\log$ (gravity) for a sample of galactic OB stars. Red triangles are dwarfs, pink pentagons are subgiants, green square are giants, yellow octagons are bright giants, blue circles are supergiants. Open symbols represent magnetic stars (with magenta heptagons being Of?p stars). Evolutionary tracks with rotation are from Ekstroem et al. (2012). Typical error bars are given. (From Martins et al. 2015.)

The combination of mass loss and rotation dramatically influences the postMain Sequence evolution of massive stars. Figure 4 shows the evolutionary tracks for non-rotating and rotating stellar models at solar metallicity (Georgy et al. 2012). We see that the upper luminosity for stars ending their lifetimes as red supergiants is between 20 and $25 M$ for non-rotating stellar models. For the rotating models, it is between 15 and $20 M_{\odot}$, quite in agreement with current observations. Without rotation, the $20 M_{\odot}$ model ends its life as a red supergiant, the $25 M_{\odot}$ as a WNL star, the 32 and $40 M_{\odot}$ as WNE stars. With rotation, the corresponding end points respectively are WNL, WNL, WC and WC stars. The most massive stars, from $60 M_{\odot}$ and above, all finish their life as WC stars. However, the rotating $60 M_{\odot}$ models make no or a smaller excursion in the LBV domain, they essentially follow a track of homogeneous evolution and directly turn to WR stars at the end of the phase of H-burning.

This different final evolution of massive stars also leads to different types of supernovae and final remnants. When a WR star explodes, one expects that a type Ib (WN) or a type Ic (WC) supernova event occurs. Interestingly, single star models with rotation, under the assumption that these stars at the end of their evolution produce these two types of supernovae, succeed in providing reasonable fit to the observed frequency of such supernovae (Georgy et al. 2012). 

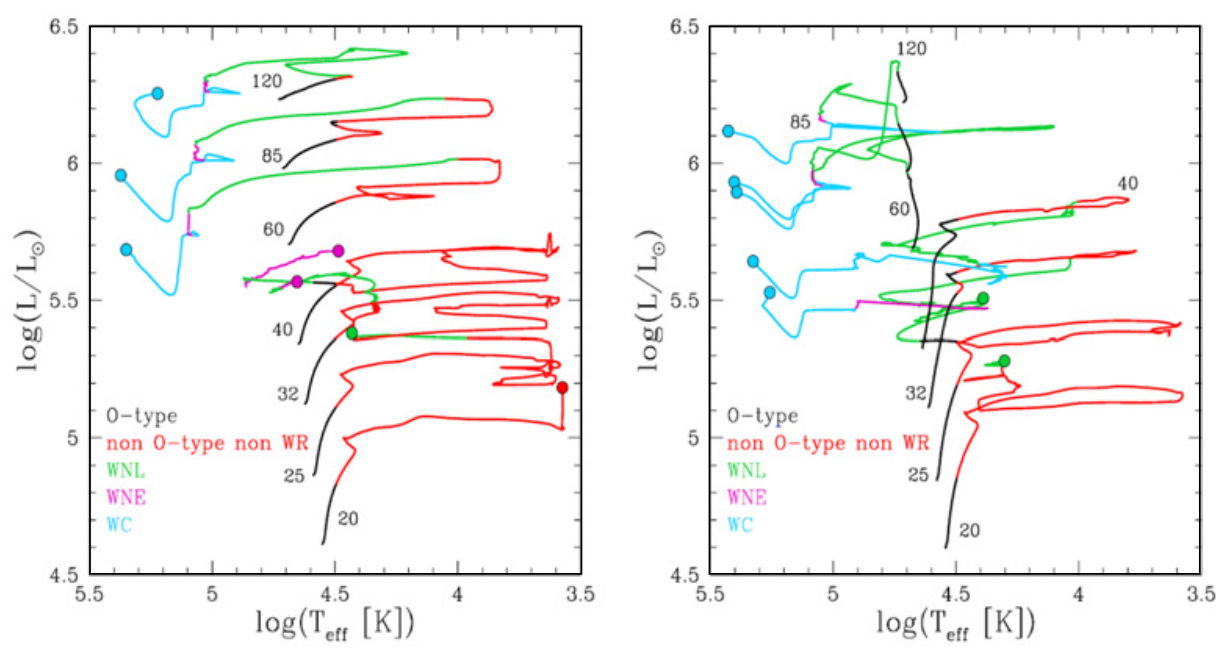

Fig. 4. HRD of the massive models from 20 to $120 M_{\odot}$ with solar composition. The different phases are marked in colors (O-type: blue; neither O-type nor WR: red; WNL: green; WNE: purple; WC: cyan). The identification of the different WR subtypes is done using mainly the surface composition, using spectral features may change a bit the situation as shown by Groh et al. (2013). Left: non-rotating models. Right: rotating models. For WR stars, the effective temperature is that at the surface of the hydrostatic core. The endpoints of the tracks are indicated by a circle. (From Georgy et al. 2012.)

\subsection{Binary stars}

Tidal interactions play an important role in close binary evolution on the values of the orbital period and of the rotation velocities (de Mink et al. 2009). New models have been performed by Song et al. (2013) accounting for tidal interactions, meridional circulation, shear instabilities and horizontal turbulence. Depending on the orbital period and the rotation velocity of the star, the tides may spin up or spin down the axial rotation of the components of the system. In both cases, tidal interaction produces a high internal differential rotation. The resulting shear mixing is so efficient that the internal distributions of angular velocity and chemical elements are strongly influenced. This produces high N- and He-enrichments at the stellar surface before any mass transfer. Figure 5 shows examples of N-enrichments. The stellar luminosities may be increased up to 0.3 dex, the evolutionary tracks and the lifetimes are modified.

Meridional circulation, when properly treated as an advection, always tends to counteract the tidal interaction, spinning up the surface when it is braked down and vice versa. As a consequence, the times needed for the axial angular velocity to become equal to the orbital angular velocity may be significantly longer than given by the typical synchronization timescale. Moreover, because of the meridional circulation some differential rotation always remains in tidally locked binary systems. 

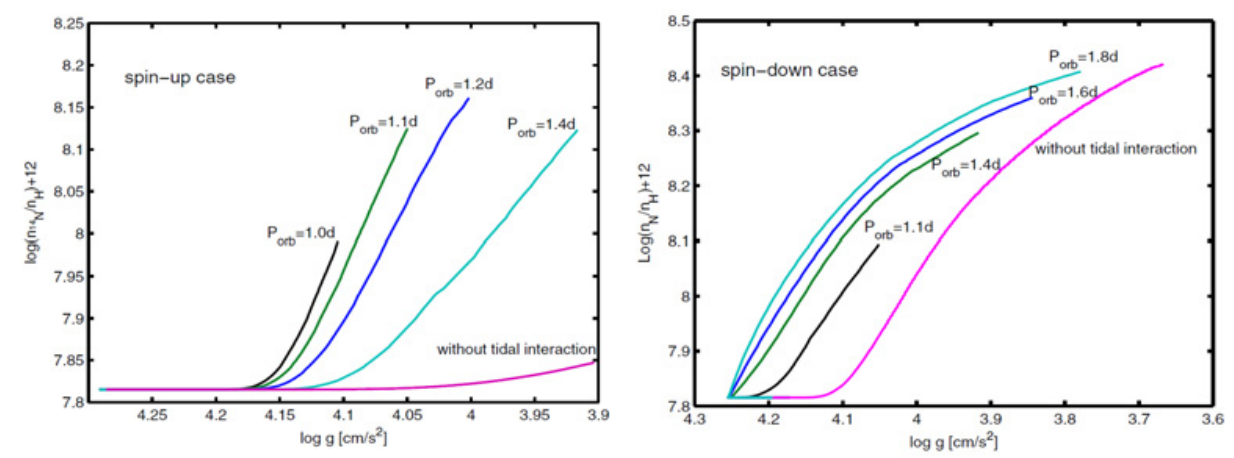

Fig. 5. Left: Variations as a function of time of the ratio of nitrogen to hydrogen (in numbers) at the surface of a $15 M_{\odot}$ stellar model with spin-up case and without tidal interactions. The curves are labeled with the values of the initial orbital period. Right: The same for spin-down. (From Song et al. 2013.)

The tidal and rotational interactions may be so high that due to mixing the primary star becomes chemically homogenous and avoids Roche lobe overflow, (homogeneous evolution means that the star evolves with a nearly uniform chemical composition from the center to the surface). Models with initial masses between 15 and $60 M_{\odot}$, for metallicities $Z$ between 0.002 and 0.014 and initial rotations equal to $30 \%$ and $66 \%$ of the critical rotation on the ZAMS are computed for stars in close binary systems by Song et al. (2016), with initial orbital periods equal to $1.4,1.6$ and 1.8 days and a mass ratio equal to $3 / 2$. The homogeneous evolution typically occurs when the rotation velocities are higher than $250 \mathrm{~km} \mathrm{~s}^{-1}$, (this velocity limit is for models with a strong coupling mediated by an internal magnetic field). If this happens, the stellar radius no longer increases during evolution and, if the stars are compact enough (especially at lower metallicity $Z$ ), they may avoid the Roche lobe overflow (RLOF) and mass transfer. The avoidance of the RLOF is favored in high mass stars (initial $M>35 M_{\odot}$ ) with lower $Z$ and short orbital periods.

We note that the homogeneous massive stars may be of interest for explaining the fast rotating Wolf-Rayet stars, the progenitors of long soft gamma ray bursts or massive binary black holes.

\section{The extremely low $Z$ stars. The case of CEMP-no stars}

A major fact about stellar rotation and evolution is that the various effects of stellar rotation are much higher at lower metallicities $Z$. There are 3 main effects responsible for that.

1. There are more fast rotators at lower $Z$, as suggested by Maeder et al. (1999) on the basis of the comparisons of the fraction of Be stars in the SMC, LMC 


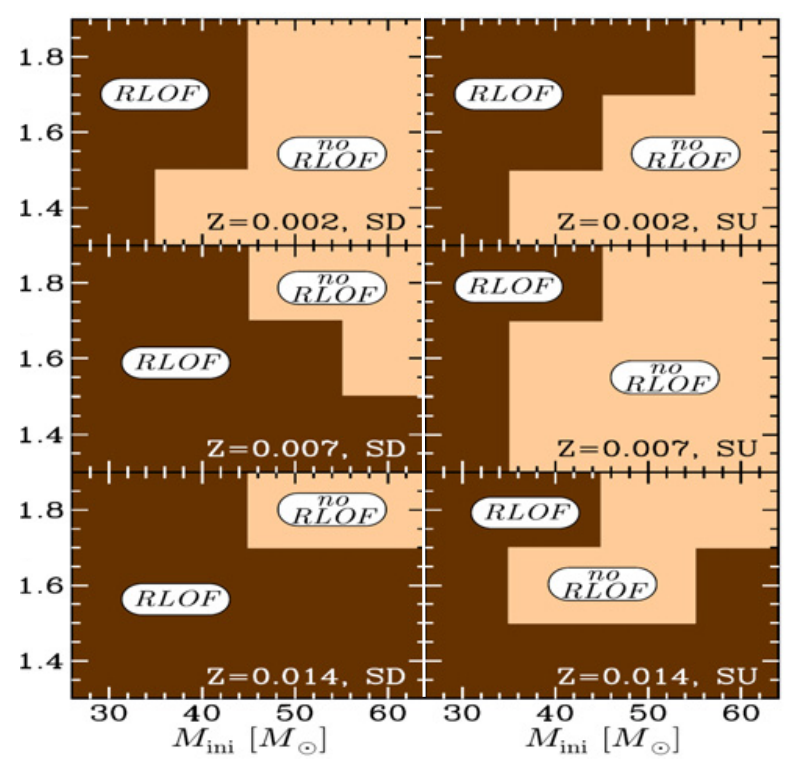

Fig. 6. The vertical axis gives the initial orbital period in days, the horizontal axis is the initial mass of the primary in a close binary system. The secondary has a mass equal to $2 / 3$ the mass of the primary. The different panels correspond to different initial metallicities $\mathrm{Z}$ and to different initial rotation of the primary. SD indicates spin-down cases, SU spin-up cases. Roche lobe overflow during the Main-Sequence phase is avoided in light reddish areas. Roche lobe overflow during the Main-Sequence phase occurs in dark brown areas. (From Song et al. 2016.)

and solar neighborhood. This was further confirmed by several subsequent authors, see for example Martayan et al. (2007).

2. A second reason came out from the Geneva models: since at lower $Z$ the stars are more compact, the internal $\Omega$-gradients are steeper and thus the shear mixing is more important. Also the timescale for mixing that is proportional to the square of the stellar radius is shorter.

3. The large surface chemical enrichments due to mixing in low $Z$ stars favors an heavy mass loss, particularly in the red supergiants, where a high C-abundance may boost the mass loss. These three effects enhance the rotational effects at lower metallicities. Several confirmations of these facts were enlightened by Chiappini (2013).

A further extreme case of rotational mixing is that of the CEMP-no stars as discussed by Maeder et al. (2015). These are Carbon Enriched Metal Poor stars with no or very little s-elements. They have the lowest known $[\mathrm{Fe} / \mathrm{H}]$ contents, typically with $[\mathrm{Fe} / \mathrm{H}]$ between -2.5 and -7 or less. Their masses are between about 0.8 and 
1.0 $M_{\odot}$ and they are generally found in the Outer Halo and in small spheroidal galaxies. These extreme objects show very large excess of carbon, up to about $[\mathrm{C} / \mathrm{Fe}]=4$, for recent references see Hansen et al. (2016). They also have large excesses of $\mathrm{N}$ and $\mathrm{O}$, as well as some excesses of $\mathrm{Na}, \mathrm{Mg}$ and $\mathrm{Al}$. These peculiarities are not due to self-enrichment, since many CEMP-no stars are still MS stars or subgiants. The peculiar abundances of CEMP-no stars reflect the yields of some star(s), the source $\operatorname{star}(\mathrm{s})$, in a previous generation, which may be the first one.

Figure 7 presents the ${ }^{12} \mathrm{C} /{ }^{13} \mathrm{C}$ vs. $[\mathrm{C} / \mathrm{N}]$ ratios for known CEMP-no stars and compares these data to those of the AGB and spinstar models. CEMP-no stars have low ${ }^{12} \mathrm{C} /{ }^{13} \mathrm{C}$ ratios providing evidence of CNO burning. However, the huge amounts of $\mathrm{C}$ (as well as $\mathrm{O}$ ) necessarily imply some synthesis of $\mathrm{C}$ and $\mathrm{O}$ by the $3 \alpha$ reaction during He-burning in the source stars. Thus, CEMP-no stars likely exhibit products of $\mathrm{He}$-burning that have gone through partial mixing and some processing by the $\mathrm{CNO}$ cycle (in the source stars), producing low ${ }^{12} \mathrm{C} /{ }^{13} \mathrm{C}$ and a broad variety of $[\mathrm{C} / \mathrm{N}]$ ratios, as suggested by Maeder et al. (2015). Partial mixing means that there were not a large number of turnovers between the $\mathrm{H}-$ and $\mathrm{He}-$ burning regions. Some $\mathrm{C}$ and $\mathrm{O}$ coming by mixing from the $\mathrm{He}-$ burning region has been turned into nitrogen, but some fractions of this $\mathrm{C}$ and $\mathrm{O}$ have not been further processed by the $\mathrm{CNO}$ cycle.

If we had full mixing as in a convective zone, the ${ }^{13} \mathrm{C}$ would be rapidly destroyed by the ${ }^{13} \mathrm{C}(\alpha, n){ }^{16} \mathrm{O}$ reaction by going into the He-burning region, leading to high ${ }^{12} \mathrm{C} /{ }^{13} \mathrm{C}$ ratios. The stars would show s-elements, resulting from neutron captures of the neutrons emitted by the reactions ${ }^{13} \mathrm{C}(\alpha, n){ }^{16} \mathrm{O}$ and ${ }^{22} \mathrm{Ne}(\alpha, n){ }^{25} \mathrm{Mg}$. As CEMP-no stars are generally characterized by a relative absence of s-elements, this is consistent with partial mixing. Then, if the outer stellar layers of the source star are ejected by stellar winds, they escape further nuclear processing. A combination of partial mixing and mass loss produces chemical yields corresponding to the kind of abundances observed in CEMP-no stars.

The model data for AGB envelopes with masses from 2 to $6 M_{\odot}$ and metallicity $Z=0.0001$ by Herwig (2004) and those by Karakas \& Lattanzio (2007) with 1 to $6 M_{\odot}$ and $Z=0.0001$ are also shown in Figure 7 . The comparison with observations reveals a large difference between CEMP-no stars and the location of AGB stars in this diagram, as already shown by Masseron et al. (2010). For all the $[\mathrm{C} / \mathrm{N}]$ values, the predicted ${ }^{12} \mathrm{C} /{ }^{13} \mathrm{C}$ ratios are generally higher than observed.

The model of spinstars has been developed to represent this kind of objects, as shown by Meynet et al. (2010) and Maeder \& Meynet (2012): these are massive stars generally of low $Z$ with fast rotation, strong mixing, and high mass loss. The mixing in spinstars, due mainly to shear instabilities, currently brings products of the CNO-burning $\left({ }^{14} \mathrm{~N}\right.$ and $\left.{ }^{13} \mathrm{C}\right)$ to the stellar surface. It may happen that products of the He-burning $(\mathrm{C}$ and $\mathrm{O})$ are mixed in H-burning regions, thus producing primary ${ }^{14} \mathrm{~N}$ and ${ }^{13} \mathrm{C}$, which may reach the stellar surface (primary means produced from the initial $\mathrm{H}$ and $\mathrm{He}$ ). During $\mathrm{H}$-burning, especially in low $Z$ models, which are hotter and denser than models at solar composition, the 


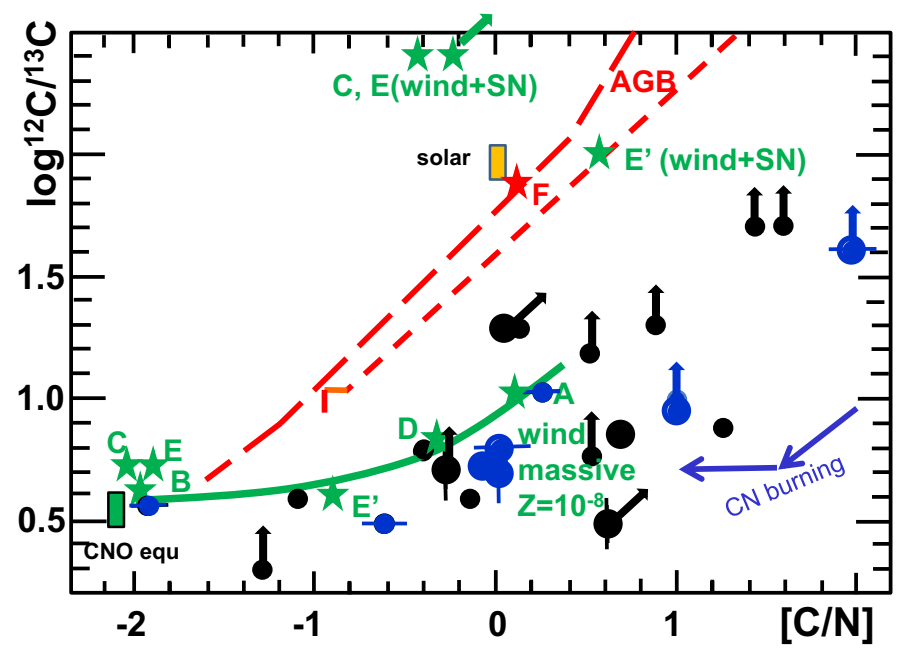

Fig. 7. Observations of ${ }^{12} \mathrm{C} /{ }^{13} \mathrm{C}$ ratios vs. [C/N] (black dots Norris et al. 2013, blue dots Masseron et al. 2010). A vertical bar indicates MS or subgiant stars with low Li content, a horizontal bar indicates CEMP-no or low-s stars by Masseron et al. which are also cataloged as "b-" by Allen et al. (2012). The big points apply to MS stars or to subgiants close to the turnoff with $T_{\text {eff }}>5500 \mathrm{~K}$ and $\log g \geq 3.25$, as by Norris et al. (2013), while the small points represent the bright giants with lower $T_{\text {eff }}$ and $\log g$. Some of the ${ }^{12} \mathrm{C} /{ }^{13} \mathrm{C}$ values are lower bounds indicated by a vertical arrow. If at the same time, the $\mathrm{N}$ value is an upper bound, the arrow is oblique. Rectangles indicate the solar and the CNO equilibrium values. AGB models with $Z=10^{-4}$ are indicated by broken red lines, the upper one from Karakas \& Lattanzio (2007), and the lower one by Herwig (2004). The low masses are on the top of the lines. A red star shows a fast-rotating AGB model of $7 M_{\odot}$ with $Z=10^{-5}$ (model F) by Meynet et al. (2010). Green stars show models of rapidly rotating massive stars A, B, C, D with masses from 40 to $85 M_{\odot}$ and $Z=10^{-8}$ from Hirschi (2007). Model $\mathrm{E}$ and $\mathrm{E}^{\prime}$ have a mass of $60 M_{\odot}$ with $Z=10^{-5}$ and are given in Table 4 by Meynet et al. (2006); they differ by the value of the mass loss rates (higher in $\mathrm{E}^{\prime}$ ). A parenthesis (wind+SN) indicates the values when both the wind and the supernova contributions are counted. The directions of CN burning are indicated.

$\mathrm{Ne}-\mathrm{Na}$ and $\mathrm{Mg}-\mathrm{Al}$ reactions (or cycles) also significantly occur involving the isotopes of these elements.

In the He-burning regions, ${ }^{22} \mathrm{Ne}$ is produced from $\alpha$-captures on ${ }^{14} \mathrm{~N}$. Further reactions may also occur in models of very low $Z$, such as the ${ }^{22} \mathrm{Ne}(\alpha, \gamma)^{26} \mathrm{Mg}$ and ${ }^{22} \mathrm{Ne}(\alpha, n){ }^{25} \mathrm{Mg}$ reactions, which produce other daughters of nitrogen. The ${ }^{16} \mathrm{O}(\alpha, \gamma){ }^{20} \mathrm{Ne}$ and the ${ }^{20} \mathrm{Ne}(\alpha, \gamma){ }^{24} \mathrm{Mg}$ reactions may also operate during He-burning in the hot conditions of low $Z$ stars. These various reactions produce elements able to reach the stellar surface and modify the compositions 
of the winds and the chemical yields of the first stellar generations. Many other plots of particular abundance ratios have been shown by Maeder et al. (2015).

The mixing processes enrich the stellar surface in elements, which like carbon, increase the opacity of the outer layers and enhance the mass loss rates of the models having initially very low $Z$. Without mixing, the models would have kept inefficient stellar winds, while with metallicity enrichment the stellar winds can reach the strength of those in the Magellanic Clouds as shown by Meynet et al. (2006) and Hirschi (2007). The consequence is that the contribution of the stellar winds to the chemical yields can be very significant in advanced evolutionary phases. We could even think that the winds may be the main source of chemical enrichment for massive stars (say above $25 M_{\odot}$ ), which end their life as a black hole with a large fallback.

Some models by Meynet et al. (2006), Meynet et al. (2010) provide the total yields, summing the wind and the supernova contributions. These models are represented in Figure 7 with a parenthesis "(wind+SN)". These results cannot account for the CEMP-no observed abundances. We notice that the lines that would connect models $\mathrm{C}, \mathrm{E}$, and $\mathrm{E}^{\prime}$ to models $\mathrm{C}$ (wind $\left.+\mathrm{SN}\right), \mathrm{E}($ wind $+\mathrm{SN})$, and $\mathrm{E}^{\prime}($ wind $+\mathrm{SN})$ have a slope of about 1.0 corresponding to the addition of ${ }^{12} \mathrm{C}$. The physical reason is clear: supernovae produce much ${ }^{12} \mathrm{C}$, while they produce little ${ }^{13} \mathrm{C}$ and ${ }^{14} \mathrm{~N}$, thus making a slope of 1.0 . This plot indicates that CEMP-no stars do not receive the complete addition of the ${ }^{12} \mathrm{C}$ layer from the source stars from the "onion skin model" during supernovae explosions.

On the whole, these examples show how critical are the rotational effects in order to understand the detailed physics of many interesting objects, and apparently even of the first stars.

\section{Where are the mysteries?}

Does it mean that everything is solved? There are still unsolved problems. Indeed, if the models of rotating stars well explain the observed chemical peculiarities of many kinds of stars, the situation is much less satisfactory concerning the predictions concerning the rotational properties of stars.

\subsection{Problems with rotational coupling}

The first case concerns the interior solar rotation. The rotational splitting of $\mathrm{p}+\mathrm{g}$ modes of solar oscillations provides the internal $\Omega$-profile of the Sun, showing an almost flat $\Omega$-curve down to the center, with an increase by only about $10 \%$ in the inner $20 \%$ of the solar radius, see Turck-Chieze et al. (1997) and references therein. The problem is that the models with current hydrodynamic effects as discussed above produce an insufficient coupling to lead to such a flat curve: the predicted $\Omega$-profile are always too steep with a much higher central solar rotation. Many solutions have been proposed, such as the effects of gravitational winds or a higher $D_{\mathrm{h}}$. Our preferred solution, for reasons given at the end of this section, 
is that of an additional coupling by internal magnetic field (Eggenberger et al. 2005), however the problem is still open.

The same problem of the lack of internal coupling also occurs for the red giants. The rotational splitting of mixed modes for red giants from Kepler provides the internal rotation profiles of low mass red giants, as shown by Beck et al. (2012), see also Deheuvels et al. (2014) among others. Again, the predicted contrast between core rotation and envelope rotation is much higher than predicted. This disagreement has raised many studies emphasizing the need of some additional internal coupling (e.g. Eggenberger et al. 2012; Eggenberger et al. 2016). The TaylerSpruit dynamo produces insufficient coupling as shown by Cantiello et al. (2014). We note that this does not prevent the possibility of a fossil magnetic field being responsible for some additional coupling. There is also difficulties to account for the low core rotation in terms of gravitational waves (Fuller et al. 2014). These authors note "The observed spin-down of cores on the red giant branch cannot be totally attributed to gravity waves, but the waves may enhance shear within the radiative region and thus increase the efficacy of viscous/magnetic torques." Belkacem et al. (2015) further precise that gravity waves give an insufficient contribution to slow down the core for subgiants, however this appears possible for evolved red giants. The missing viscosity should increase with evolution as shown by Cantiello et al. (2014) and Eggenberger et al. (2016).

Remarkably, the same problem is present for the white dwarfs. They show rotational velocities $v \sin i$ lower than 2 to $10 \mathrm{~km} / \mathrm{s}$, which are 1 to 2 orders of magnitude lower than predicted (Berger et al. 2005). The inclusion of the TaylerSpruit dynamo does not solve the problem (Suijs et al. 2008), despite the claim of the authors, the discrepancy being still by an order of a magnitude.

Again, the pulsar rotation shows the same kind of effect as found all the way of stellar evolution. The rotation in the advanced stages of massive stars was studied by Hirschi et al. (2004) and by Heger et al. (2005). An interesting result is that, while during the evolution from the Main Sequence to the pre-supernova stage, the central $\Omega$ increases by 4 orders of magnitude, the central specific angular momentum changes by less than one order. The rotation period of the $2.7 M_{\odot}$ core with a density of about $10^{8} \mathrm{~g} \mathrm{~cm}^{-3}$ of an initial $25 M_{\odot}$ star is of the order of 1 sec. At the pulsar birth with a density of $10^{14} \mathrm{~g} \mathrm{~cm}^{-3}$, the rotation period should be about $0.1 \mathrm{~ms}$, assuming angular momentum conservation with $\Omega \sim \varrho^{2 / 3}$. But, it is generally considered that pulsars at birth have a rotation period of about $10 \mathrm{~ms}$. There may be some loss during the explosive pulsar formation, however such an important loss may have occurred in previous evolution. Thus, through the whole stellar evolution as well as for different masses, there seems to be some additional internal coupling that reduces the central rotation.

\subsection{The role of magnetic field}

The masses and the evolutionary stages, for which there are some evidences of an additional coupling producing a slowing down of stellar cores, are very different. These masses and stages involve very different timescales. We find it unlikely that 
the observed discrepancies are due to some drastic changes of the current rather slow hydrodynamical processes, such as meridional circulation and mixing, especially more that the mixing of the chemical elements is relatively well accounted for throughout the evolution.

We suggest that the effects of a fossil magnetic field, whether sustained or not by some dynamos in various cases, may be responsible for the missing coupling of the cores and envelopes at various stages. This is all the more possible since from the Main Sequence to the end stages, like white dwarfs and pulsars, the magnetic fields seems to be more or less compatible with magnetic flux conservation. In a first step in this direction, it has been shown by Maeder \& Meynet (2014) that the direct magnetic coupling of the core to the extended convective envelope of red giants and supergiants appears unlikely. However, the intermediate radiative zone is fully coupled to the core during the He-burning and later phases. This coupling is able to produce a strong spindown of the core of red giants and supergiants, also leading to relatively slowly rotating stellar remnants such as white dwarfs and pulsars.

\section{Conclusions}

Our dear and so much regretted friend Jean-Paul has shown us the way to the understanding of rotational mixing in stars. His major contributions will stand forever, they have led to impressive developments in stellar evolution. Now, the progress of observations brings new challenges and his successors have to go on in the same spirit of understanding the deep physics of stellar evolution.

\section{References}

Allen, D.M., Ryan, S.G., Rossi, S., et al., 2012, A\&A, 548, A34

Beck, P.G., Montalban, J., Kallinger, T., et al., 2012, Nature, 481, 55

Belkacem, K., Marques, J.P., Goupil, M.J., et al., 2015, A\&A, 579, A31

Berger, L., Koester, D., Napiwotzki, R., et al., 2005, A\&A, 444, 565

Cantiello, M., Mankovich, C., Bildsten, L., et al., 2014, ApJ, 788, 93

Chaboyer, B. \& Zahn, J.-P., 1992, A\&A, 253, 17

Chiappini, C., 2013, Astron. Nachr., 334, 595

Deheuvels, S., Dogan, G., \& Goupil, M.J., 2014, A\&A, 564, 27

de Mink, S.E., Cantiello, M., \& Langer, N., et al., 2009, A\&A, 497, 243

Eggenberger, P., Maeder, A., \& Meynet, G., 2005, A\&A, 440, L9

Eggenberger, P., Montalban, J., \& Miglio, A., 2012, A\&A, 544, L4

Eggenberger, P., Lagarde, N., \& Miglio, A., et al., 2016, AN, 337, 83

Ekstroem, S., Georgy, C., \& Eggenberger, P., 2012, A\&A, 537, 146

Fuller, J., Lecoanet, D., \& Cantiello, M., et al., 2014, ApJ, 796, 17

Georgy, C., Ekstroem, S., \& Meynet, G., et al., 2012, A\&A, 542, A29

Groh, J.H., Georgy, C., \& Ekström, S., 2013, A\&A, 558, L1

Hansen, C.J., Nordstroem, B., \& Hansen, T.T., 2016, A\&A, 588, 37 
Heger, A., Woosley, S.E., \& Spruit, H.C., 2005, ApJ, 626, 350

Herwig, F., 2004, ApJS, 155, 651

Hirschi, R., Meynet, G., \& Maeder, A., 2004, A\&A, 425, 649

Hirschi, R., 2007, A\&A, 461, 571

Karakas, A., \& Lattanzio, J.C., 2007, PASA, 24, 103

Maeder, A., 2009, Physics, Formation and Evolution of Rotating Stars (Sringer Verlag, Berlin Heidelberg), p. 829

Maeder, A., Grebel, E.K., \& Mermilliod, J.-C., 1999, A\&A, 346, 459

Maeder, A., \& Meynet, G., 2012, Rev. Mod. Phys., 84, 25

Maeder, A., \& Meynet, G., 2014, ApJ, 793, 123

Maeder, A., Meynet, G., \& Chiappini, C. 2015, A\&A, 576, 56

Maeder, A., Meynet, G., Lagarde, N., \& Charbonnel, C., 2013, A\&A, 553, 1

Maeder, A., \& Zahn, J.-P., A\&A, 334, 1000

Martayan, C., Frémat, Y., \& Hubert, A.-M., et al., 2007, A\&A, 462, 683

Martins, F., Herv, A., \& Bouret, J.-C., et al., 2015, A\&A, 575, A34

Masseron, T., Johnson, J.A., \& Plez, B.A., 2010, A\&A, 509, A93

Meynet, G., \& Maeder, A., 1997, A\&A, 321, 465

Meynet, G., Ekstroem, S., \& Maeder, A., 2006, A\&A, 447, 623

Meynet, G., Hirschi, R., \& Ekstroem, S., et al., 2010, A\&A, 521, A30

Norris, J.E., Yong, D., \& Bessel, M.S., et al., 2013, ApJ, 762, 28

Przybilla, N., Firnstein, M., \& Nieva, M.F., et al., 2010, A\&A, 517, 38

Song, H.F., Maeder, A., Meynet, G., Huang, R.Q., Ekstroem, S., \& Granada, A., 2013, A\&A, 556, 100

Song, H.F., Meynet, G., Maeder, A., Ekstroem, S., \& Eggenberger, P., 2016, A\&A, 585, 120

Suijs, M.P.L., Langer, N., \& Poelarends, A.-J., et al., 2008, A\&A, 481, L87

Talon, S., \& Zahn, J.-P., 1997, A\&A, 317, 749

Turck-Chieze, S., Palacios, A., Marques, J.P., \& Nghiem, P.A.P., 2010, ApJ, 715, 1539

Zahn, J.-P., A\&A, 265, 115 